\title{
Comparison of Multi-Detector Computed Tomography (MDCT) and 3 Tesla Magnetic Resonance Imaging (MRI) in Evaluation of Orbital mass lesions
}

\author{
Urmil Chawla ${ }^{1}$, Rohtas K. Yadav ${ }^{2}$, Vandana Sharma ${ }^{3 *}$, Nithin Narayanan ${ }^{4}$ \\ ${ }^{1}$ Professor, Regional Institute of Ophthalmology, PGIMS, Rohtak, Haryana, India \\ ${ }^{2}$ Sr. Professor, Dept. of Radiodiagnosis, PGIMS, Rohtak, Haryana, India \\ ${ }^{3}$ Assistant Professor, Dept. of Ophthalmology, Adesh Institute of Medical Sciences and Research, Bathinda, Punjab, India \\ ${ }^{4}$ Junior Resident, Dept. of Radiodiagnosis, PGIMS, Rohtak, Haryana, India
}

\begin{abstract}
DOI: $10.36348 /$ sjmps.2021.v07i01.011
| Received: 05.01.2021 | Accepted: 15.01.2021 | Published: 21.01.2021
\end{abstract}

*Corresponding author: Dr. Vandana Sharma

\section{Abstract}

The study was done to compare results of different imaging modalities Computed Tomography (CT scan) and Magnetic Resonance Imaging (MRI) with operative or histopathological diagnosis and to determine the accuracy of each. Forty patients with clinically or ultrasonographically diagnosed intraorbital masses were enrolled in the study who did not have any contraindication for contrast enhanced CT or MRI. A correct diagnosis was made in 37 patients and an incorrect diagnosis in 3 patients on CT scan while correct diagnosis was made on MRI in 38 patients and incorrect diagnosis was made in 2 patients. The clinical, surgical and histopathological findings were used as the gold standard for making a final diagnosis in these patients. There was an excellent agreement between CT and MRI in predicting extraorbital involvement, intracranial extension and optic nerve involvement. CT predicted bony involvement and areas of calcification better whereas MRI was better in predicting extraocular muscle involvement.

Keywords: Magnetic resonance imaging, Computed Tomography, orbit, contrast.

Copyright (C) 2021 The Author(s): This is an open-access article distributed under the terms of the Creative Commons Attribution 4.0 International License (CC BY-NC 4.0) which permits unrestricted use, distribution, and reproduction in any medium for non-commercial use provided the original author and source are credited.

\section{INTRODUCTION}

The advent of ultrasonography (USG) in medical imaging revolutionized the imaging of orbit. It became the first choice of investigation for orbital pathologies due to its lack of radiation, widespread availability and ability to characterize certain lesions. The addition of duplex and colour Doppler ultrasonography has added more value to the same. It is now widely used for evaluation of posterior segment of the eyeball and part of retrobulbar space, but it has its limitations in diagnosing retrobulbar pathology, painful orbital lesions and imaging the origin and extension of masses. Also, operator dependence, lack of specificity and poor characterization and localization of lesions makes it inferior in a number of conditions [1].

To overcome the limitations of USG, came Computed Tomography scan (CT scan) with the ability to identify and diagnose orbital mass lesions, inflammatory changes and bony abnormalities. Continuous scanning protocols in the latest models provide a physiological switch from $2 \mathrm{D}$ to $3 \mathrm{D}$ (dimensional) volumetric acquisition and improved Zaxis resolution. Faster scanning helps in reducing the motion artifacts significantly. This is especially advantageous in the evaluation of old subjects, children and sick patients. However, CT is less useful (compared to MRI) for the evaluation of pathologies of optic nerve sheath complex, visual pathways and orbital apex where there is little orbital fat because of its poor softtissue contrast resolution. Iodinated contrast is relatively contraindicated in patients with allergy, previous history of severe reactions and severe renal failure. Additionally, dense calcifications, metallic foreign bodies and bony areas may produce beam hardening artefacts [2-5].

Although initially considered expensive and non-reliable for orbital lesions, MRI has gained popularity as it produces images without ionizing radiation, has excellent contrast resolution and can obtain images in any plane without changing the position of the patient. Indications for MRI include suspected orbital tumours, lesions of the optic nerve especially at the apex, tumours with potential intracranial spread and visualization of the cavernous sinus. Since bone is poorly visualized on MRI, its utilization in trauma is limited. Also, certain contraindications such as metallic intraocular foreign 
Urmil Chawla et al., Saudi J Med Pharm Sci, Jan, 2021; 7(1): 54-59

body, cardiac pacemaker, aneurysm clips etc.; artificial motion due to lid movement, tattoo/cosmetics, low spatial resolution and claustrophobia limit its utility [24].

This study was planned to diagnose, characterize and define the extent of various orbital mass lesions, to differentiate between benign and malignant lesions and to correlate the findings of MDCT and 3T MRI with operative or histopathological findings and clinical follow-up.

\section{Material ANd Method}

A total of 40 patients from all age groups, of either sex were enrolled in the study after taking a written informed consent. The study design was cleared by the Institutional Ethics Committee and was in accordance with the tenets of Declaration of Helsinki. The subjects enrolled had orbital mass lesions diagnosed clinically and/or on ultrasonography. A detailed history and a thorough ophthalmological examination was performed.

Ultrasonography and X-ray of orbits and skull were done in required cases. Patients with a history of orbital trauma, any contraindication to CECT and CEMRI such as allergy to contrast agent or acute or chronic renal failure, patients unable to undergo MRI due to claustrophobia, ferromagnetic foreign bodies within the globe and metallic implants, pacemakers, aneurysmal clips etc. were excluded from the study.

All Multi detector CT examinations were done on Siemens Somatom Volume Zoom plus 4 spiral CT scanner. Axial thin sections were done on plain CT scan and then followed by coronal and sagittal reconstruction of images. A non-ionic low osmolar water-soluble contrast medium with a concentration of $350-400 \mathrm{mg}$ iodine $/ \mathrm{ml}$ was given to patients to visualize the vascular structures of the orbit as well as to know the pattern of enhancement of lesions. Contrast of $50 \mathrm{ml}$ for adults and $2 \mathrm{ml} / \mathrm{kg}$ body weight for children was used. CT of the brain and paranasal sinuses was also done wherever required.

MRI examinations were performed using Discovery MR750w GE 3 Tesla strength. MRI protocol for examination of the orbit included the following planes and sequences

- Axial: T1 WI, T2 WI and SPIR T1 WI

- Coronal: T1 WI and T2 WI

- Sagittal: T1 WI

- Post-Gd: axial T1WI, coronal T1WI, sagittal T1WI and SPIR T1WI

- Diffusion-Weighted MRI

Other MRI sequences were done as and when required. Gadolinium contrast agents were given as intravenous bolus injection in a dose of about $10 \mathrm{ml}$ for adults and $1 \mathrm{ml} / 5 \mathrm{~kg}$ body weight for children.

The imaging modalities were compared based on the different imaging features. The kappa $(\mathrm{k})$ value was provided where statistically feasible. Kappa values less than zero were considered negative correlation. According to Landis and Koch's classification scale, kappa statistics of less than 0.40 indicated poor agreement beyond chance, statistics in the range of $0.41-0.75$ indicated fair to good prognosis beyond chance and statistics greater than 0.75 indicated excellent agreement beyond chance.

After radiological examination, data and various findings on MDCT and 3T MRI were collected and results were tabulated. The clinical profile of the patient was taken into consideration while making a diagnosis or a differential diagnosis on each modality. The diagnosis was compared with the final diagnosis made on surgery/biopsy/FNAC/FNAB/clinically or by therapeutic follow-up. The diagnosis was considered correct if it was present in the list of differential diagnosis of that imaging modality. The relevant statistical analysis was then done.

\section{RESULTS}

The age of the patients in the present study ranged from 5 days to 80 years with an average age of 33.86 years. Most of the patients were in the first decade of life signifying the fact that children and young adults had more incidences of orbital masses with another peak in the late decades. There was a male preponderance with 23 males and 17 females being enrolled in the study. The left eye was involved in 18 patients, the right eye was involved in 14 patients and bilateral eyes were involved in 8 patient.

Most patients had proptosis (45\%) as the chief complaint. The next most common symptom was visible orbital swelling, present in 10 patients. Pain and redness were present in 6 and 4 patients respectively. Pain along with redness was present in 2 patients. In 5 patients, there was blurring/loss of vision. Growth on the lid was present in 3 patients. Leukocoria was present in 2 patients.

Of the 40 patients who underwent CT and MRI evaluation, 39 patients $(97.5 \%)$ had a mass lesion in their orbit. No definite mass lesion could be made out in 1 patient $(2.5 \%)$ on CT or MRI. As for location of mass lesions, 11 lesions $(27.5 \%)$ were found in the extraconal region of the orbit and $10(25 \%)$ in the intraconal region of the orbit. (Table 1) Most of the patients in the study had regular margins of the mass lesion $23(57.5 \%)$. Calcification was observed in 7 $(17.5 \%)$ cases on CT. However, MRI could not detect calcification in any of the lesions. 
Table-1: Site of orbit involved in mass lesion

\begin{tabular}{|l|l|l|}
\hline SITE OF ORBIT INVOLVED & No.of cases(n) & Percentage \\
\hline Extraconal & 11 & 27.5 \\
\hline Intraconal & 10 & 25 \\
\hline Superolateral orbit & 7 & 17.5 \\
\hline Globe & 6 & 15 \\
\hline Preseptal & 4 & 10 \\
\hline Panorbital & 2 & 5 \\
\hline
\end{tabular}

Most of the mass lesions were iso to hyperdense $(n=15)$ on CT images followed by hyperdense lesions in 12 patients $(30 \%)$. Ten $(25 \%)$ lesions were hypodense in appearance while 3 (7.5\%) lesions showed fat on CT.

On examining the MRI, 13 lesions (32.5\%) were isointense on T1W images, whereas 29 lesions $(72.5 \%)$ were hyperintense on $\mathrm{T} 2 \mathrm{~W}$ images. On fatsuppressed contrast-enhanced T1 weighted images, 36 (90\%) lesions were hyperintense in appearance. Out of 40 study patients, 19 (47.5\%) showed diffusion restriction on diffusion-weighted imaging. Remaining $21(52.5 \%)$ showed no diffusion restriction. 33(82.5\%) patients showed contrast enhancement on CT and $35(87.5 \%)$ patients showed contrast enhancement on
MRI, out of which 3 patients showed delayed and heterogeneous enhancement. Equal no. of lesions i.e. $6(15 \%)$ of the total 40 lesions showed intracranial extension on both CT and MRI.

Various imaging features of orbital mass lesions were compared on both CT and MRI. There was an excellent agreement between CT and MRI in the visualization of all margins of lesions, optic nerve involvement, intracranial extension and extraorbital extension in terms of the kappa coefficient. However, CT was more /sensitive in the detection of calcification in the lesion and involvement of the adjacent bone. MRI predicted the involvement of extraocular muscles more accurately (Table 2).

Table-2: Comparison between CT and MRI in evaluation of orbital masses

\begin{tabular}{|c|c|c|c|}
\hline Findings & $\begin{array}{l}\text { No. of patients on } \\
\text { CT (n) }\end{array}$ & $\begin{array}{l}\text { No. of patients } \\
\text { on MRI (n) }\end{array}$ & $\begin{array}{l}\text { Kappa coefficient } \\
0-0.40=\text { Poor } \\
0.40-0.75=\text { Good } \\
>0.75=\text { Excellent }\end{array}$ \\
\hline Visualisation of all margins & 40 & 40 & 1 \\
\hline Calcification & 7 & 0 & 0 \\
\hline Optic nerve involvement & 10 & 10 & 1 \\
\hline Extraocular muscle involvement & 13 & 15 & 0.890 \\
\hline Bone involvement & 11 & 8 & 0.795 \\
\hline Intracranial extension & 6 & 6 & 1 \\
\hline Extraorbital extension & 11 & 11 & 1 \\
\hline Contrast enhancement & 33 & 35 & 0.805 \\
\hline
\end{tabular}

\section{BASIS OF FINAL DIAGNOSIS}

The final diagnosis was made in $35(87.5 \%)$ patients by surgery or other invasive procedures like excision of mass in 26(65\%), FNAC in 4(10\%), debulking in $2(5 \%)$, Trucut biopsy in $2(5 \%)$ and surgical drainage and debridement in 1(2.5\%) lesion. Remaining 5 patients $(12.5 \%)$ were followed up clinically and the final diagnosis was made by the clinical response (Table 3).

Table-3: Basis of final diagnosis of patient

\begin{tabular}{|l|l|l|}
\hline PROCEDURE DONE & NO.OF PATIENTS & PERCENTAGE \\
\hline Excision biopsy & 26 & 65 \\
\hline Follow up & 5 & 12.5 \\
\hline FNAC & 4 & 10 \\
\hline Debulking & 2 & 5 \\
\hline Trucut biopsy & 2 & 5 \\
\hline Surgical drainage and debridement & 1 & 2.5 \\
\hline
\end{tabular}

Based on CT findings, the correct diagnosis was made in $37(92.5 \%)$ patients and an incorrect diagnosis was made in $3(7.5 \%)$ patients. The correct diagnosis was made on MRI in $38(95 \%)$ patients and incorrect diagnosis was made in $2(5 \%)$ patients (Table 4).Surgery or other invasive procedures were done in 
Urmil Chawla et al., Saudi J Med Pharm Sci, Jan, 2021; 7(1): 54-59

$35(87.5 \%)$ patients. The surgical and histopathological findings were used as the gold standard for making a final diagnosis in these patients. Remaining patients $(n=5,12.5 \%)$ were followed up clinically and the clinical response was taken as the criteria for making the final diagnosis.

Table-4: Comparison of imaging diagnosis/differential diagnosis with the final diagnosis

\begin{tabular}{|l|l|l|l|}
\hline Final diagnosis & No. of cases & CT diagnosis & MRI diagnosis \\
\hline Optic nerve glioma & 2 & ++ & ++ \\
\hline Meibomian gland carcinoma & 1 & + & + \\
\hline Retinal hemangioblastoma & 1 & + & + \\
\hline Pleomorphic adenoma of lacrimal gland & 2 & ++ & ++ \\
\hline Haemangioma & 3 & +-+ & +-+ \\
\hline Microphthalmos with cyst & 1 & + & + \\
\hline Lipoma & 1 & + & + \\
\hline Melanoma & 4 & +++- & ++++ \\
\hline Cysticercosis & 1 & + & + \\
\hline Chondroid syringoma & 1 & - & - \\
\hline Thyroid orbitopathy & 2 & ++ & ++ \\
\hline Dermoid cyst & 2 & ++ & ++ \\
\hline Inflammatory mucocele & 1 & + & + \\
\hline Neurofibroma & 1 & + & + \\
\hline Meningioma & 1 & + & + \\
\hline Metastasis & 3 & +++ & +++ \\
\hline Venolymphatic malformation & 3 & +++ & +++ \\
\hline Lymphoma & 3 & +++ & +++ \\
\hline Retinoblastoma & 1 & + & + \\
\hline Inflammatory mass & 2 & ++ & ++ \\
\hline Phpv & 1 & + & + \\
\hline Adenoid cystic carcinoma of lacrimal gland & 1 & + & + \\
\hline Sinonasal adenocarcinoma & 1 & + & + \\
\hline Pituitarymacroadenomawith orbital apex involvement & 1 & + & + \\
\hline
\end{tabular}

+ Correct diagnosis and/or differential diagnosis, - Incorrect (not included in differential diagnosis)
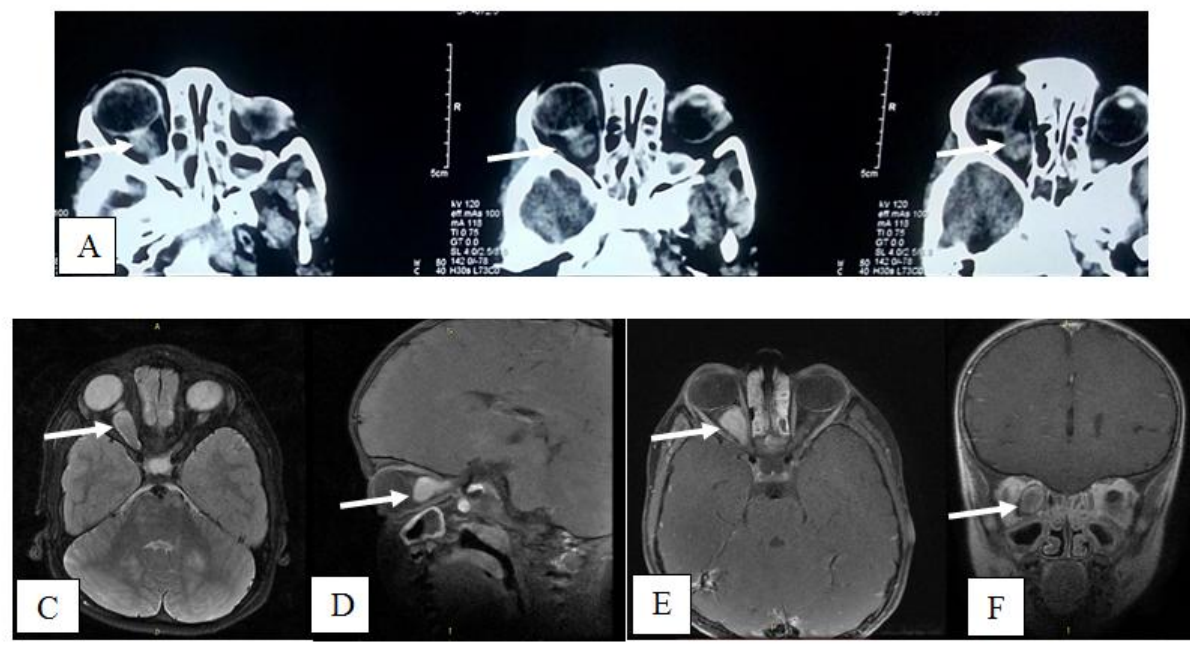

Fig-1: (A) show a well-defined, fusiform mass lesion with regular margins in the retrobulbar orbit, arising from the intraconal part of the optic nerve which is hyperdense on axial NCCT images with homogeneous contrast enhancement on CECT. Fig (C), (D), (E) AND (F) shows a welldefined mass lesion with regular margins which is hyperintense on T2 weighted MR images in intraconal part of the optic nerve in the same patient. The lesion is showing intense homogeneous contrast enhancement on gadolinium administration. On histopathological examination, optic nerve glioma was confirmed.

\section{DiSCUSSION}

The diagnosis of orbital disease was one of the most challenging aspects of ophthalmology before the advent of Computed Tomography (CT) and Magnetic Resonance Imaging (MRI). A multi-modality imaging approach is required for orbital mass lesions. In our prospective study, we tried to study the spectrum and imaging features of various intraorbital masses causing proptosis on CT and MRI. We also assessed the efficacy of imaging in the characterization of intraorbital masses by correlating with pathological diagnosis wherever possible and tried to standardize the imaging algorithm for evaluation of intraorbital masses. 
Urmil Chawla et al., Saudi J Med Pharm Sci, Jan, 2021; 7(1): 54-59

In our study, CT and MRI detected a welldefined mass lesion in 39 patients and an ill-defined lesion in 1 patient. However, in a study conducted by Forbes et al. [6] CT could detect 133/137(96\%) orbital neoplasms. Also, Berges et al. [7] who evaluated 140 orbital masses described the detection of $139 / 140$ (99.3\%) orbital masses with CT scan. This is contrary to our study in which CT detected all the 40(100\%) of the lesions. This difference can be explained by the use of older generation CT scanners in these studies, which could have led to decreased sensitivity in the detection of orbital lesions.

It was also observed that contrast enhancement in both CT and MRI was a good predictor of the vascularity of the mass. Although, contrast enhancement is not a reliable factor to distinguish benign lesions from malignant ones, it was seen more often in malignant and inflammatory mass lesions. This is in accordance with the finding by Ben Simon et al. [8], who studied 131 patients with biopsy-proven orbital tumours and described that contrast enhancement on MRI scan was more prevalent in malignant and inflammatory lesions.

MRI was superior to $\mathrm{CT}$ in demonstrating the extraocular muscle involvement which was noted in $15(37.5 \%)$ lesions. Although both the modalities were equally effective in depicting the course of the optic nerve in our study, MRI was better in showing it separate from the mass lesions in $30(75 \%)$ patients. This is because the bony structure of the optic canal interferes in direct visualization of the optic nerve in CT scan while it is seen devoid of any signal on MRI [9, 10].

CT was found to be good in predicting the involvement of bone in the form of widening of the optic nerve canal or lateral margin of orbit, remodelling of the lesser wing of sphenoid, a sclerotic expansile lesion and cortical breach or lytic destruction. On the contrary, a study by Edward et al. [11] had shown equal sensitivity of CT and MRI in evaluating bone involvement.

Therefore, to summarize, there was an excellent agreement between CT and MRI in predicting extraorbital involvement, intracranial extension and optic nerve involvement. CT was better in predicting bony involvement and was more sensitive in the detection of calcification whereas MRI was better in predicting extraocular muscle involvement.

The major limitation of our study was a small sample size $(n=40)$. Comparison between imaging and intraoperative findings was possible only in those 35 patients who had undergone any invasive procedure such as surgical excision or FNAC. Remaining 5 patients $(12.5 \%)$ were followed up clinically and the final diagnosis was made by the clinical response. Also, the pathological spectrum was very heterogeneous with many of the histopathological groups having a single patient each.

\section{CONCLUSION}

MRI must be considered the imaging modality of choice for anatomical delineation, accurate diagnosis and a preoperative assessment of spread, vascularity of the lesion in a patient who is suspected to have an intraorbital mass. CT scan may be directly done as a sole modality, if MRI is not available, not affordable or if there are contraindications for MRI. CT could also be done additionally to supplement the MRI findings if there is suspicion of bone involvement or calcification.

\section{REFERENCES}

1. Vijayvargiya, R., \& Shukla, A. (2017). Role of MRI in Evaluation of Orbital Mass Lesions with Ultrasonographic and Histopathological Correlation. International Journal of Medical Research Professionals, 3, 156-68.

2. Massoud, T.F., \& Cross, J.J. (2008). Grainger and Allison's Diagnostic Radiology 5th Ed., Churchill Livingstone.

3. Nesi, F. A., Lisman, R. D., \& Levine, M. R. (1998). Smith's ophthalmic plastic and reconstructive surgery. American Journal of Ophthalmology, 4(125), 569-570.

4. Dreyfus, Heran, F. (2017). Imaging an Orbital Mass: The Essential. Journal of the Belgian Society of Radiology, 101(S1), 2. DOI: http://doi.org/10.5334/jbr-btr.1429.

5. Sharma, O.P. (2005). Orbital sonography with its clinic-surgical correlation. Indian Journal of Radiological Imaging, 15, 537-54.

6. Forbes, G. S., Earnest, F., 4th, \& Waller, R. R. (1982). Computed tomography of orbital tumors, including late-generation scanning techniques. Radiology, 142(2), 387-394. https://doi.org/10.1148/radiology.142.2.7054827

7. Berges, O., Vignaud, J., \& Aubin, M. L. (1984). Comparison of sonography and computed tomography in the study of orbital space-occupying lesions. AJNR. American journal of neuroradiology, 5(3), 247-251.

8. Ben Simon, G. J., Annunziata, C. C., Fink, J., Villablanca, P., McCann, J. D., \& Goldberg, R. A. (2005). Rethinking orbital imaging establishing guidelines for interpreting orbital imaging studies and evaluating their predictive value in patients with orbital tumors. Ophthalmology, 112(12), 2196-2207.

https://doi.org/10.1016/j.ophtha.2005.09.013

9. Kornreich, L., Blaser, S., Schwarz, M., Shuper, A., Vishne, T. H., Cohen, I. J., Faingold, R., Michovitz, S., Koplewitz, B., \& Horev, G. (2001). Optic pathway glioma: correlation of imaging findings with the presence of neurofibromatosis. 
Urmil Chawla et al., Saudi J Med Pharm Sci, Jan, 2021; 7(1): 54-59

AJNR. American journal of neuroradiology, 22(10), 1963-1969.

10. Listernick, R., Darling, C., Greenwald, M., Strauss, L., \& Charrow, J. (1995). Optic pathway tumors in children: the effect of neurofibromatosis type 1 on clinical manifestations and natural history. The Journal of pediatrics, 127(5), 718-722. https://doi.org/10.1016/s0022-3476(95)70159-1
11. Edwards, J. H., Hyman, R. A., Vacirca, S. J., Boxer, M. A., Packer, S., Kaufman, I. H., \& Stein, H. L. (1985). 0.6 T magnetic resonance imaging of the orbit. AJR. American journal of roentgenology, 144(5), 1015-1020.

https://doi.org/10.2214/ajr.144.5.1015 